\title{
A Study of the Extremum of the Total Energy of the Selective Signals Constructed by Quadratic Splines
}

\author{
Irina Strelkovskaya', Irina Solovskaya' ${ }^{1,2}$, Anastasiya Makoganiuk ${ }^{1 *}$ \\ 1 Department of Higher Mathematics, Education and Research Institute for Infocommunication and Software Engineering, \\ O.S. Popov Odessa National Academy of Telecommunications, Kuznechnaya St. 1, Odessa, Ukraine \\ 2 Department of Switching Systems Education and Research Institute for Infocommunication and Software Engineering, \\ O.S. Popov Odessa National Academy of Telecommunications, Kuznechnaya St. 1, Odessa, Ukraine \\ *Corresponding author, e-mail: a.makoganyuk@onat.edu.ua
}

Received: 25 April 2018, Accepted: 23 October 2018, Published online: 20 December 2018

\begin{abstract}
The development of mobile communication networks in the direction of $5 \mathrm{G}$ networks implies the use of the radio interface that is based on new signal-code structures, the choice of which will determine their further development and the ability of operators to provide innovative services. The use of quadratic splines for the synthesis of selective signals with a finite spectrum, free from intersymbol interference, is proposed. An analytical expression for the synthesized signal in the time and frequency domains is obtained. A study was made of the dependence of the total energy of a selective signal on the parameters of a quadratic spline, which is used to interpolate the spectral density in the transition region using the methods of differential calculus of functions of several variables. To study the extremum of the total energy of a selective signal, the parameters of the width of the transition area a was used and the coefficient of rounding the spectrum $\rho$, the variation of whose spectral density allowed us to establish the limits of the change in the total energy of the signal in question. Conducted studies allow us to synthesize the signal and formulate recommendations on how by changing the parameters of the signal, you can get a signal with the desired properties. This will allow to obtain the optimal waveform in accordance with the selected criteria, providing for the required energy performance of the signal in the radio interface of $5 \mathrm{G}$ networks.
\end{abstract}

Keywords

selective signal, spectral density, the total energy of the signal

\section{Introduction}

The development of mobile networks today is in the direction $5 \mathrm{G}$ networks, the implementation of which will significantly enhance the ability of the mobile operator to provide innovative high-speed services, including tactile Internet services, transmission of holographic images, industrial and transportation automation with support for low-value time delay of packets in the network. Significant changes in the network structure based on software-configured networks in the SDR (Software Defined Radio) radio access network and the SDN (Software Defined Networking) core network are required for such features of the network operation, as well as support for the virtualization procedures of network functions and new radio access technologies.

The main work associated with the standardization of fifth-generation $5 \mathrm{G}$ networks [1-4] suggests the possibility of creating a broadband mobile access eMBB (Extreme

Mobile Broadband) based on new types of signal-code designs. Their use should increase the transmission rate up to $20 \mathrm{Gbit} / \mathrm{s}$ network $5 \mathrm{G}$ and the spectral efficiency of up to $30 \mathrm{bit} / \mathrm{s} / \mathrm{Hz}$, as well as to achieve reduction of the delay time of packets. This will provide a wider range of IoT (Internet of things) services and M2M machine-to-machine interaction, such as Video Surviliance, IoT-cameras, smart-M2M objects, Smart house, Smart parking IoT, Smart grids and, in the long term, holographic TV, virtual VR services and augmented AR realities. It is the choice of the type of signal that will be used at the radio interface $5 \mathrm{G}$ networks, depends on their further development and the possibility of operators to provide necessary services. Today, a number of signal-code constructions, each of which has significant features in implementation has been proposed by the world's leading equipment manufacturers. At the same 
time, the question of choosing a method and criteria for evaluating the effectiveness of each solution remains open. The considered tasks of technological development of the radio interface of $5 \mathrm{G}$ networks include the need to study the energy characteristics of signals in order to determine the optimal waveform. The obtained results will allow making an informed choice of an effective signal for various conditions of its use and implementation.

The works of the authors [5-13] are devoted to the study of various signal constructions, in which methods are proposed for studying the characteristics of signals in the time-frequency domain, which make it possible to use them for existing signals. One of the methods for studying the characteristics of the signals proposed in [5] is the use of cross-correlation and autocorrelation functions, which allow us to estimate the efficiency of spectrum use. In work [6], the use of various types of wavelet functions for estimating the frequency characteristics of signals was considered, but this approach is most appropriate for a group of complex signals. The work [7] is devoted to the study of the channel performance extremum, in which the extremum is in accordance with the error probability criterion. The authors used spline functions in works [8-18], that can significantly improve the interpolation accuracy, while simplifying the procedure for mathematical calculations. Today it is difficult or even impossible to specify such a class of problems where the spline-interpolation methods would not find practical application. These methods are effective in problems of optimization, interpolation, control, and many others. The main advantages of spline interpolation include:

1. splines are more stable with respect to local perturbations, that is, the behavior of a spline in the vicinity of a point does not affect the behavior of the spline as a whole, as, for example, this occurs with polynomial interpolation;

2. good convergence of spline interpolation in contrast to polynomial. In particular, for functions with irregular smoothness properties, it is advisable to use spline interpolation.

In addition to these properties, splines have useful extreme properties. For example, splines are solutions to minimization problems for functionals that estimate the smoothness of functions in the Hilbert norm and in the norm of the space of bounded functions, which greatly simplifies the solution of many practical problems.

A method of interpolation of the spectral characteristics of selective signals based on cubic splines was proposed by authors in works [8-10], that allows to synthesize new signal functions, the extreme properties of which improve the technical characteristics of network facilities. A comparative analysis of the possibility of using polynomial splines, B-splines and cubic splines for interpolation of signals was carried out in works [11-12] and the main advantages, methods and positions of interpolation are described in these works. However, the search for new methods of interpolation of spectral characteristics selective signal in the present conditions of the active technology is an urgent task. It is interesting to solve the problem of interpolation of the spectral density of the considered selective signals using another type of spline functions, for example, quadratic, which will reduce the computational aspect when finding the analytical form of the synthesized signal and its total energy. Earlier, when determining the extremal properties of selective signals based on quadratic splines, the authors used the criterion of the magnitude of the aperture of the eye diagram and the D-criterion of the change in the total energy of the selective signal [14].

In this work, it is required to find the extremum of the total energy of a selective signal constructed on the basis of quadratic splines using the differential calculus of functions of several variables. As a signal for the study of energy indicators, we use a selective signal with a finite spectrum that satisfies the first Nyquist criterion and is characterized by the absence of intersymbol interference [8-9]. It is known that the appearance of intersymbol interference is a consequence of distortions and fading in the radio interface, therefore its increase leads, first of all, to a reduction in noise immunity and losses. Minimizing the intersymbol interference due to implementation of a special type of signal structures allows to provide a certain level of quality characteristics, e.g., the probability of erroneous reception symbols. In addition, when implementing radio interface technologies in $5 \mathrm{G}$ mobile communication networks, it is rather important to solve the problem of using the frequency resource and reducing the level of mutual interference. Summarizing the above, it can be argued that the search for new methods of interpolation of the spectral characteristics of signals and the study of their extreme properties is an urgent task.

The purpose of this work is to study the dependence of the total energy of a selective signal on the parameters of a quadratic spline, which is used to interpolate the spectral density in the transition region to improve the signal quality characteristics. 


\section{Study of the complete energy of selective signal}

2.1 Synthesis of selective signals using quadratic splines Consider a selective signal with a finite spectrum whose signal function satisfies the first Nyquist criterion [4], if in the time domain it is equal to:

$$
g(k T)=\left\{\begin{array}{l}
U \text { for } k=0, \\
0 \text { for } k= \pm 1, \pm 2, \ldots,
\end{array}\right.
$$

where $T$ is the duration of the clock interval (symbol duration), $U$ - value of the instantaneous value of the pulse at the moment of counting $t=0$.

The main property of the selective signals follows directly from the expression Eq. (1) - the presence of equidistant zeros in the time domain, and hence the complete absence of intersymbol interference (ISI). Thus, the signals that satisfy the first Nyquist criterion take the value $U$ at $t=0$, and at equidistant points that follow at intervals of duration $T$, take zero values. Such signals are also called selective or reference [8-9]. Their main property, which distinguishes them from the signals of other classes, is the absence of intersymbol interference.

For a more detailed study of the effect of the shape of the spectrum $G(j \omega)$ on the behavior of the signal function $g(t)$ in the intervals between equidistant zeros, we represent the spectral density in the form $[2,3]$

$$
|G(j \omega)|= \begin{cases}U T, & |\omega|<\omega_{A}, \\ G_{\Delta 1}(\omega), & \omega_{A} \leq|\omega|<\omega_{C}, \\ G_{\Delta 2}(\omega), & \omega_{C}<|\omega| \leq \omega_{B}, \\ 0, & \omega_{B}<|\omega|,\end{cases}
$$

where $U=g(0), T$ is the duration of the clock interval, $\omega_{A}=(1-\alpha) \omega_{C} ; \omega_{B}=(1+\alpha) \omega_{C} ; \omega_{C}=\pi / T ; \alpha=\left(\omega_{C}-\omega_{A}\right) / \omega_{C}$ - the coefficient of the rounding of the spectrum, which determines the width of the transition region $\left[\omega_{A}, \omega_{B}\right]$ $(0 \leq \alpha \leq 1), \Delta \omega=\alpha \omega_{C}$ (Fig. 1). It is known that the properties of the selective signals, in particular, their behaviour in the gaps between the equidistant zeros are closely related to the shape of their spectral density Eq. (2). Therefore, we consider the features of these relationships and the effect of approximations of the signal function in this area.

According to $[2,3]$, the functions $G_{\Delta 1}(\omega)$ and $G_{\Delta 2}(\omega)$ are related by expression

$$
G_{\Delta 1}(\omega)=U T-G_{\Delta 2}\left(2 \omega_{C}-\omega\right), \omega_{A} \leq|\omega| \leq \omega_{C}
$$

The analytical expression for the spectral density in the transition region $\left[\omega_{A}, \omega_{B}\right]$ can be obtained by using the method of interpolation by quadratic spline [19-20]. Interpolating quadratic spline on $\left[\omega_{C}, \omega_{B}\right]$, and then, using

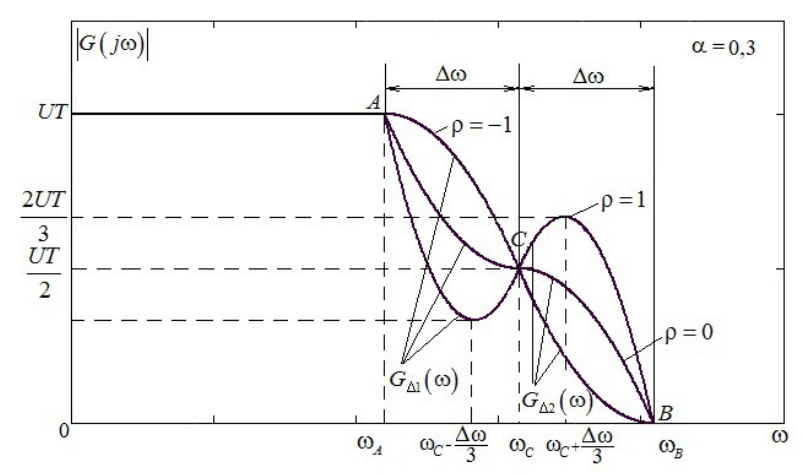

Fig. 1 Interpolation of the spectrum on the basis of a quadratic spline

Eq. (3), it will be possible to restore the shape of the function $|G(j \omega)|$ in the transition region.

As a result of interpolation of the spectral density by a quadratic spline [5], we obtain

$$
G_{\Delta 2}(\omega)=\frac{U T}{2}+\frac{\rho U T}{\Delta \omega}\left(\omega-\omega_{C}\right)-\frac{U T(1+2 \rho)}{2(\Delta \omega)^{2}}\left(\omega-\omega_{C}\right)^{2},
$$

where $\rho$ is found from relation

$G_{\Delta 2}^{\prime}\left(\omega_{C}\right)=\frac{\rho U T}{\Delta \omega}$.

The inverse Fourier transform of the spectral density Eq. (2), taking into account Eq. (3) and Eq. (4), makes it possible to obtain an analytical expression for the selective signal $g(t)$. However, it is easier to use the expressions obtained in [9].

According to [5], the analytic expression of the selective signal $g(t)$ has the form

$$
g(t)=2 U \frac{\sin \omega_{C} t}{\omega c^{t}}\left[\frac{(1+\rho) \sin (\Delta \omega t)}{\Delta \omega t}-\frac{(1+2 \rho)(1-\cos (\Delta \omega t))}{(\Delta \omega)^{2} t^{2}}\right]
$$

and the total energy of the signal $g(t)$

$$
E_{\omega}(\alpha, \rho)=U^{2} T\left[1+\frac{\alpha}{30}\left(2 \rho^{2}-3 \rho-12\right)\right]
$$

The greatest specificity that affects the time-frequency characteristics of the signal has a transition region, which represents a transition from a maximum value of the normalized spectral density of zero. The behaviour of a function in a given area affects intersymbol distortions, the redistribution of energy in the signal function, and other characteristics of the signals themselves. Therefore, the optimization of the spectral characteristics in the transition domain affects all the quality indicators of the signals and, ultimately, the characteristics of the quality of QoS. 


\subsection{Study on the total energy extremum selective signals that are based on quadratic splines}

To study the extremum of the total energy of the selective signal from the parameters $\alpha$ and $\rho$, we determine the range of admissible values of the parameters $\alpha$ and $\rho$. The main characteristic of the transition region is its width, characterized by the parameter $\alpha$, which is the coefficient of rounding the spectrum. The parameter $\rho$ characterizes the shape of the spectrum in the transition region.

The parameter $\alpha$ varies from 0 to 1 , that is, $0 \leq \alpha \leq 1$. It is necessary to find within what limits the parameter $\rho$ varies. Taking into account that Eq. (2) is a nonnegative function, and taking into account odd symmetry with respect to point $\mathrm{C}$, we can write the inequality:

$0 \leq G_{\Delta 2}(\omega) \leq U T, \quad \omega_{C} \leq \omega \leq \omega_{B}$.

With increasing $\rho$, the function $G_{\Delta 2}(\omega)$ approaches the line $U T$ and at some frequency $\rho=\rho_{\max }$ and it touches this line at the frequency $\omega=\omega_{M}$. To determine $\rho_{\max }$ and $\omega_{\max }$, we form two equations, the first of which has the form:

$0.5+\frac{\rho}{\Delta \omega}\left(\omega_{M}-\omega_{C}\right)-\frac{1+2 \rho}{2(\Delta \omega)^{2}}\left(\omega_{M}-\omega_{C}\right)^{2}=1$.

To obtain the second one, we equate the derivative of the function $G_{\Delta 2}(\omega)$ of the form Eq. (4) to zero. We have $\frac{\rho U T}{\Delta \omega}-\frac{1+2 \rho}{(\Delta \omega)^{2}}\left(\omega_{M}-\omega_{C}\right)=0$.

Consider Eq. (9). We introduce a new variable $x=\omega_{M}-\omega_{C}$, then

$x=\frac{\rho \Delta \omega}{1+2 \rho}$.

To find $\rho_{\max }$, we substitute Eq. (10) into Eq. (8), we obtain

$0.5+\frac{\rho}{\Delta \omega} x-\frac{1+2 \rho}{2(\Delta \omega)^{2}} x^{2}=1$.

When solving the quadratic Eq. (11), we have

$\rho_{1}=1+\sqrt{2} \approx 2.414, \rho_{2}=1-\sqrt{2} \approx-0.414$.

Studies show that when $\rho_{1}=1+\sqrt{2} \approx 2.414$ $G_{\Delta 2}(\omega)$ reaches a maximum at $\left[\omega_{C} ; \omega_{B}\right]$, and for $\rho_{2}=1-\sqrt{2} \approx-0.414$ the extremum of the function $G_{\Delta 2}(\omega)$ is outside the interval $\left[\omega_{C} ; \omega_{B}\right]$. In this way $\rho_{\text {max }}=1+\sqrt{2} \approx 2.414$.

Define $\omega_{M}$ for $\rho_{\max } \approx 2.414$ from the relation $x=\omega_{M}-\omega_{C}=\frac{\rho_{\max } \Delta \omega}{1+2 \rho} \quad$ or $\quad \omega_{M}=\frac{\rho_{\max } \Delta \omega}{1+2 \rho}+\omega_{C}$.
Let us find the minimum allowable value of the parameter $\rho$. At $\rho=\rho_{\text {min }}$, the curve function $G_{\Delta 2}(\omega)$ becomes a parabola that touches the $\omega$ axis at point B with coordinates $\left((1+\alpha) \omega_{C}, 0\right)$. We make the change of variable $x=\omega_{M} / \omega_{C}$. Taking into account that $\Delta \omega=\alpha \omega_{C}$, we investigate the roots of the following quadratic equation:

$\frac{-1+2 \rho}{2\left(\Delta \omega^{2}\right)}\left(\omega_{M}-\omega_{C}\right)^{2}+\frac{\rho}{\Delta \omega}\left(\omega_{M}-\omega_{C}\right)+0.5=0$,

$\frac{1+2 \rho}{2 \alpha^{2}}(x-1)^{2}-\frac{\rho}{\alpha}(x-1)-0.5=0$,

$(1+2 \rho) x^{2}+x(-2-4 \rho-2 \alpha \rho)+1+2 \rho+2 \alpha \rho-\alpha^{2}=0$.

Rewrite Eq. (12) in the form

$a x^{2}+b x+c=0$

where

$a=1+2 \rho, b=-2-4 \rho-2 \alpha \rho, c=1+2 \rho+2 \alpha \rho-\alpha^{2}$.

Represent Eq. (13) in the form

$a(x-v)^{2}=0$

or

$a x^{2}-2 x v a+a v^{2}=0$.

Equating the coefficients of the same powers in Eq. (13) and Eq. (14), we obtain the system of equations

$\left\{\begin{array}{l}b=-2 a v, \\ c=a v^{2} .\end{array}\right.$

It follows directly from Eq. (15)

$v=\frac{-b}{2 a}=\frac{2 \rho+1+\alpha \rho}{1+2 \rho}$.

Then Eq. (14) can be rewritten

$(1+2 \rho)\left[x-\left(1+\frac{\alpha \rho}{1+2 \rho}\right)\right]^{2}=0$.

A parabola $y=-(1+2 \rho)\left[x-\left(1+\frac{\alpha \rho}{1+2 \rho}\right)\right]^{2}$ will have a root of multiplicity 2 at the point $x_{B}=1+\alpha$ if the equality $\frac{\rho}{1+2 \rho}=1$ holds. Hence $\rho_{\min }=-1$. Thus, the range of admissible values of $D$ parameters $\alpha$ and $\rho$ has the form of a rectangle (Fig. 2):

$D=\{(\alpha, \rho): 0 \leq \alpha \leq 1,-1 \leq \rho \leq 1+\sqrt{2}\}$.

Let us study the dependence of the total energy of the selective signal on its parameters in the domain $D$. 
And find the largest and smallest values that are achieved in this area.

To do this, consider the function

$$
f(\alpha, \rho)=E_{\omega}(\alpha, \rho) / U^{2} T .
$$

We have

$$
f(\alpha, \rho)=1+\frac{\alpha}{30}\left(2 \rho^{2}-3 \rho-12\right) \text {. }
$$

To determine the extremum points of the function $f(\alpha, \rho)$, we find its partial derivatives

$f_{\alpha}^{\prime}(\alpha, \rho)=\frac{1}{30}\left(2 \rho^{2}-3 \rho-12\right), f_{\rho}^{\prime}(\alpha, \rho)=\frac{\alpha}{30}(4 \rho-3)$,

that exist everywhere in the domain $D$.

Taking advantage of the necessary conditions for an extremum function of two variables [6], we find the stationary points by solving the system of equations

$\alpha=0, \rho_{1}=\frac{3+\sqrt{105}}{4} \approx 3.312, \rho_{2}=\frac{3-\sqrt{105}}{4} \approx-1.812, \rho=\frac{3}{4}$,

$\left\{\begin{array}{l}\frac{1}{30}\left(2 \rho^{2}-3 \rho-12\right)=0, \\ \frac{\alpha}{30}(4 \rho-3)=0,\end{array} \Leftrightarrow\left\{\begin{array}{l}2 \rho^{2}-3 \rho-12=0, \\ \frac{\alpha}{30}(4 \rho-3)=0,\end{array}\right.\right.$

that does not correspond to the region of admissible values of the parameter $\rho$. Consequently, there are no extremum points in $D$. We find the smallest and largest value of the function $f(\alpha, \rho)$ on the boundary of the domain $D$ (Fig. 1).

If function Eq. (7) is differentiable in a restricted closed domain, then it reaches its maximum or minimum value either at a stationary point (this is the point at which the derivative vanishes or does not exist) or at the boundary point of the region. In this case, the function is the function under study Eq. (17), and the region is the domain $D$ (Fig. 2).

In solving this problem, stationary points were found and studies were conducted at stationary points and it was shown that stationary points are not included in the considered domain $D$. We conclude that in the domain $D$ there are no extremum points, therefore it remains to find the largest and smallest value of the function on the boundary of the domain $D$. This is a rectangle

$D=\{(\alpha, \rho): 0 \leq \alpha \leq 1,-1 \leq \rho \leq 1+\sqrt{2}\}$.

That is, it is necessary to investigate the function $f(\alpha, \rho)$ at the boundary of the domain $D$ in the section $M_{1} M_{2}$ Eq. (18), in the section $M_{2} M_{3}$ Eq. (20), in the section $M_{3} M_{4}$ Eq. (22) and in the section $M_{3} M_{4}$ Eq. (22).

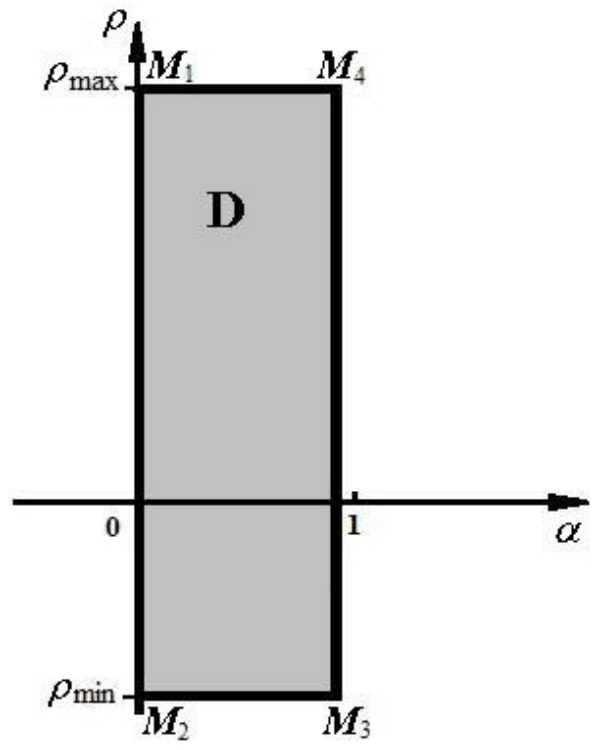

Fig. 2 The domain of the function $f(\alpha, \rho)$

In the section $M_{1} M_{2}$ (Fig. 2), we have $\alpha=0$, $\rho \in\left[\rho_{\min } ; \rho_{\max }\right]$. Then the function Eq. (17) takes the form $f(\alpha, \rho)=f(\rho)=1$.

On the section $M_{2} M_{3}$ we have $\rho_{\text {min }}=-1, \alpha \in[0,1]$. In this case Eq. (17) takes the form

$f(\alpha, \rho)=1-\frac{7 \alpha}{30}$.

By calculating $f_{\alpha}^{\prime}=-\frac{7}{30}$, we have $f_{\alpha}^{\prime}<0$ for $\alpha \in[0,1]$. Therefore, $f(\alpha)$ is a monotonically decreasing function and the smallest value on the segment $[0,1]$ takes for $\alpha=1$ and is equal to $f(1)=\frac{23}{30} \approx 0.77$; the largest is for $\alpha=0$ and is equal to $f(0)=1$. In this way,

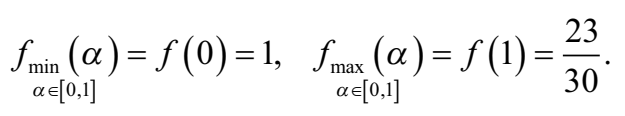

On the section $M_{3} M_{4}$, we have $\alpha=1, \rho \in\left[\rho_{\min } ; \rho_{\max }\right]$. Then the function Eq. (17) takes the form

$$
f(\alpha, \rho)=f(\rho)=1+\frac{1}{30}\left(2 \rho^{2}-3 \rho-12\right) .
$$

Calculating $f_{\rho}^{\prime}=\frac{1}{30}(4 \rho-3)$, we obtain $f_{\rho}^{\prime}=0$ for $\rho=\frac{3}{4}$. As $f_{\rho}^{\prime \prime}=\frac{4}{30}>0$, then $\rho=\frac{3}{4}$ the minimum point for the function Eq. (21). Consequently, the smallest value of the function Eq. (17) on the segment $M_{3} M_{4}$ takes at the point $M_{0}\left(1 ; \frac{3}{4}\right)$ and is equal to

$f\left(1 ; \frac{3}{4}\right)=\frac{9}{16} \approx 0.5625$. 
On the section $M_{1} M_{4}$ we have $\alpha \in[0,1]$ and $\rho=\rho_{\max }=1+\sqrt{2}$. The function Eq. (17) is represented in the form

$$
f(\alpha, \rho)=f\left(\alpha, \rho_{\max }\right)=f(\alpha)=1+(-9+\sqrt{2}) \frac{\alpha}{30} .
$$

Calculating the derivative of a function $f(\alpha)$ of the form Eq. (23), we obtain

$f_{\alpha}^{\prime}=-\frac{-9+\sqrt{2}}{30}<0$.

Consequently, at $\alpha \in[0,1]$ a function $f(\alpha)$ decreases monotonically and reaches its maximum value at $\alpha=0$. It is equal to 1 . The smallest value is achieved when $\alpha=1$. It is approximately equal to 0.747 . In this way

$$
f_{\max }(\alpha)=f(0)=1, \quad f_{\substack{\min \\ \alpha \in[0,1]}}(\alpha)=f(1)=\frac{21+\sqrt{2}}{30} \approx 0.747 .
$$

Comparing the values of Eq. (18), Eq. (20), Eq. (22), Eq. (24), we conclude that the largest value of a function of the form Eq. (17) is attained on the section $M_{1} M_{2}$, it is equal to 1 .

The smallest value of the function Eq. (17) is attained at the section $M_{3} M_{4}$ takes at the point $M_{0}\left(1 ; \frac{3}{4}\right)$ and equals $\frac{9}{16}$.

The form of the function $f(\alpha, \rho)$ is shown in Fig. 3 by the normalized magnitude of the total energy of the selective signal in three-dimensional space.

\section{Conclusion}

1. The obtained relations make it possible to calculate the energy indicators of the telecommunication system at the design stage and determine the optimal waveform in accordance with the chosen criterion.

\section{References}

[1] International Telecommunication Union "M.2083-0, Recommendation ITU-R, 2015-09, Framework and overall objectives of the future development of IMT for 2020 and beyond", 2015. [online] Available at: https://www.itu.int/dms_pubrec/itu-r/rec/m/R-RECM.2083-0201509-I!!PDF-R.pdf [Accessed: 09 October 2015]

[2] Tikhvinskiy, V., Bochechka, G. Minov, A., Babyn, A. "Razvitiye arkhitektury setey 5G" (Development of architecture 5G networks), Connect, 1-2, pp. 52-58, 2017. (in Russian) [online] Available at: http://www.niitc.ru/upload/medialibrary/75a/75abd3a9ad8ff491f452047d4f85b990.PDF [Accessed: 31 March 2017]

[3] 3GPP "Study on Scenarious and Requirements for Next Generation Access Technologies", ETSI TR 138913 V14.2.0, 2017. [online] Available at: https://www.etsi.org/deliver/etsi_tr/138900_138999/13 8913/14.02.00_60/tr_138913v140200p.pdf [Accessed: 05 May 2017]

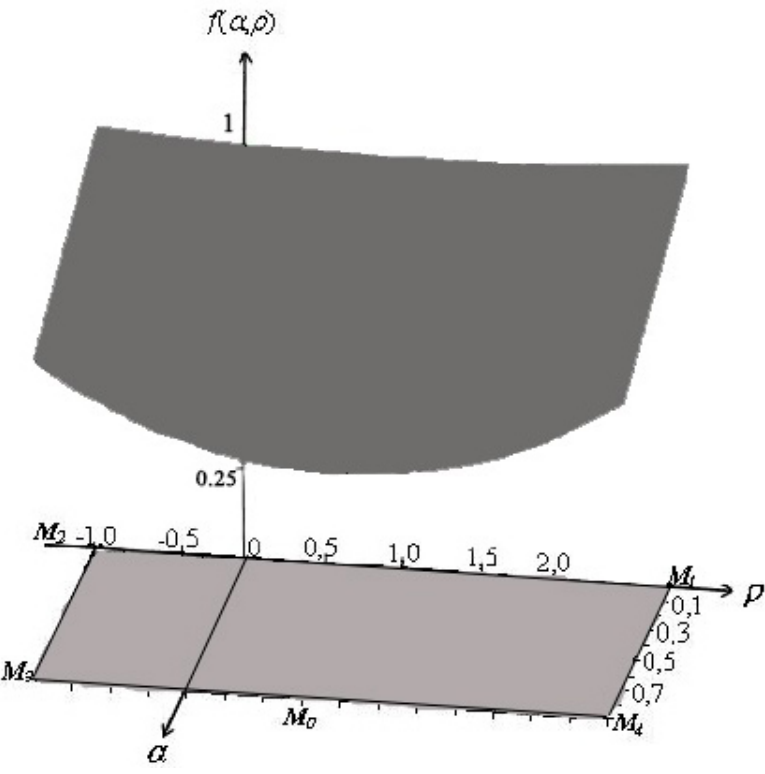

Fig. 3 The domain of the function $f(\alpha, \rho)$

So, choosing $\alpha=0, \rho \in\left[\rho_{\min } ; \rho_{\max }\right]$, where $\rho_{\min }=-1$, $\rho_{\text {max }}=1+\sqrt{2} \approx 2.414$ we obtain maximum energy of the selective signal, which is equal to 1 .

Therefore, in order to reach the maximum energy of the selective signal, it is necessary to take infinitesimals as $\alpha$.

2. Synthesized selective signals based on quadratic functions have the same properties as synthesized signals based on cubic splines. However, the calculation process of finding the synthesized signal constructed using quadratic splines less than the synthesis of selective signals on the basis of cubic splines.

3. When designing advanced radio access networks is possible, the proposed method is used to synthesize new signal functions, the extreme properties of which will improve the technical characteristics of the radio link facilities.

[4] 3GPP "Study on Architecture for Architecture for Next Generation System", TR 23.799 V14.0.0, 2016. [online] Available at: https://portal.3gpp.org/desktopmodules/Specifications/SpecificationDetails. aspx?specificationId=3045 [Accessed: 08 August 2017]

[5] Shumkiv, M., Lysechko, V., Gumenyk, A. "Statystychna otsinka metodu monitorynhu spektru na osnovi tsyfrovoyi uz'hodzhenoyi filtratsiyi" (Statistical evaluation of the spectrum monitoring method based on digital coherent filtration), Information control systems in the railway transport, 5, pp. 71-74, 2014. (in Ukrainian) [online] Available at: https:// www.google.com/url?q=http://www.irbis-nbuv.gov.ua/cgi-bin/ irbis_nbuv/cgiirbis_64.exe\%3FC21COM\%3D2\%26I21DBN\%3DUJRN\%26P21DBN\%3DUJRN\%26IMAGE_FILE_ DOWNLOAD\%3D1\%26Image_file_name\%3DPDF/ 
Ikszt_2014_5_15.pdf\&sa $=$ U\&ved $=0$ ahUKEwjj8_bDnYbfAhWDiywKHSCGCBgQFggEMAA\&client=internal-uds-cse\&cx$=012955353415694750662$ :ac0q9yn5xfi\&usg=AOvVaw3Xn0ntgg1ArF6ypnwJxZUc [Accessed: 05 May 2014]

[6] Yur, T. "Metod vyznachennya chastotnykh kharakterystyk syhnalivza dopomohoyu veyvletiv" (Method of assignment of frequency characteristics of signals for additional wavelets), System processing information, 11(136), pp. 79-86, 2015. (in Ukrainian) [online] Available at: https://www.google.com/url?q=http:// www.irbis-nbuv.gov.ua/cgi-bin/irbis_nbuv/cgiirbis_64.exe $\% 3 \mathrm{~F}-$ C21COM\%3D2\%26I21DBN\%3DUJRN\%26P21DBN\%3DUJRN\%26IMAGE_FILE_DOWNLOAD\%3D1\%26Image _ fi 1 e $\_$a m e \% 3 D P D F / s o i__ $2015{ }_{-} 11_{-} 21$. pdf\&sa=U\&ved=0ahUKEwjVzPKilYbfAhWMjCwKHfBkC8EQFggOMAU\&client $=$ internal-uds-cse $\& c x=012955353415694750662$ :ac0q9yn 5x fi\&usg=AOvVaw1j89NGPVR7HV2wrgSTpmK[Accessed: 21 November 2015]

[7] Uryvskiy, L., Procopenko, E., Vergun, S. "Issledovaniye ekstremumov proizvoditel'nosti v diskretnykh kanalakh svyazi s kodirovaniyem" (Investigation of performance extremums in discrete communication channels with coding), Naukov's notes of the Ukrainian science and research institute, 5(33), pp. 20-26, 2014. (in Russian) [online] Available at: https://www.google.com/url?q=http:// www.irbis-nbuv.gov.ua/cgi-bin/irbis_nbuv/cgiirbis_64.exe $\% 3 \mathrm{~F}-$ C21COM\%3D2\%26I21DBN\%3DUJRN\%26P21DBN\%3DUJRN\%26IMAGE_FILE_DOWNLOAD\%3D1\%26Image_ f i 1 e $\_$a m e \% 3 DP D F/ N z undiz_ $2014_{-} 55_{-} 5$. pdf\&sa $=$ U\&ved $=0$ ahUKEwi5tcS9kIbfAhVKFywKHaFiADUQFggGMAE\&client $=$ internal-uds-cse\&cx $=012955353415694750662$ :ac0q9yn5xfi\&usg=AOvVaw2caFqZHmxQQEuvGyfUmE8O [Accessed: 05 May 2014]

[8] Sukachev, E., Strelkovskaya, I. "Ekstremal'nyye svoystva selektivnykh signalov pri interpolyatsii ikh spektrov kubicheskimi splaynami" (Selective signals extremum properties in their spectra interpolation by cubic splines), Izvestiya Vysshikh Uchebnykh Zavedenij. Radioelektronika, 47(1), pp. 32-37, 2004. (in Russian)

[9] Strelkovskaya, I. V. "Application of Cubic B-Splines for Synthesis of Selective Signals", Telecommunications and Radio Engineering, 66(12), pp. 1047-1056, 2007.

https://doi.org/10.1615/TelecomRadEng.v66.i12.10

[10] Ferrer-Arnau, L., Reig-Bolaño, R., Marti-Puig, P., Manjabacas, A., Parisi-Baradad, V. "Efficient cubic spline interpolation implemented with FIR filters", International Journal of Computer Information Systems and Industrial Management Application, 5, pp. 98-105, 2013. [online] Available at: https://upcommons. upc.edu/bitstream/handle/2117/16993/Paper85.pdf [Accessed: 10 February 2017]
[11] Kim, T.-W., Kvasov, B. "A shape-preserving approximation by weighted cubic splines", Journal of Computational and Applied Mathematics, 236(17), pp. 4383-4397, 2012. https://doi.org/10.1016/j.cam.2012.04.001

[12] Pan, Z., Chen, W., Jiang, Z., Tang, L., Liu, Y., Liu, Z. "Performance of global look-up table strategy in digital image correlation with cubic B-spline interpolation and bicubic interpolation", Theoretical and Applied Mechanics Letters, 6(3), pp. 126-130, 2016. https://doi.org/10.1016/j.taml.2016.04.003

[13] Strelkovskaya, I., Makoganiuk, A., Paskalenko, S. "Comparative analysis of signal functions, built on the basis of quadratic and cubic splines", In: 2015 Second International Scientific-Practical Conference Problems of Infocommunications Science and Technology (PIC S\&T), Kharkiv, Ukraine, 2015, pp. 173-176. https://doi.org/10.1109/INFOCOMMST.2015.7357305

[14] Zakharov, Y. V., Tozer, T. C., Adlard, J. F. "Polynomial spline-approximation of Clarke's model", IEEE Transactions on Signal Processing, 52(5), pp. 1198-1208, 2004. https://doi.org/10.1109/TSP.2004.826159

[15] Sakai, M., Usmani, R. "Quadratic Spline and Two-Point Boundary Value Problem", Publications of the Research Institute for Mathematical Sciences Kyoto University, 19, pp. 7-13, 1983.

[16] Bosner, T., Rodina, M. "Variable degree polynomial splines are Chebyshev splines", Advances in Computational Mathematics, 38(2), pp. 383-400, 2011. https://doi.org/10.1007/s10444-011-9242-Z

[17] Zavyalov, Yu. S., Kvasov, B. I., Miroshnichenko, B. I. "Metody splayn-funktsiy" (Methods of spline functions), Science, Moscow, Russia, 1980. (in Russian)

[18] Ahlberg, J. H., Nilson, E. N., Walsh, J. L. "The Theory of Splines and Their Applications: Mathematics in Science and Engineering: A Series of Monographs and Textbooks, Vol. 38", 1st ed., Academic Press, NY, USA, 1967.

[19] Fikhtengolts, G. M. "Osnovy matematicheskogo analiza" (Fundamentals of mathematical analysis), Nauka, Moscow, Russia, 1968. (in Russian) 